\title{
Centrality Issues In Asymmetric Collisions: Direct Photons To The Rescue?
}

\author{
Gabor David** \\ Stony Brook University \\ E-mail: david@bnl.gov
}

\begin{abstract}
Methods to classify events experimentally according to collision geometry are well established and non-controversial when collisions of large ions are studied. However, high luminosity data from $p / d+\mathrm{A}$ collisions at RHIC and LHC provided some surprising results that either call for new physics or question the applicability of the established methods of event classification for them. So far there is no consensus in the community what is the proper model and procedure to determine centrality, how to connect observed event activity with collision geometry in very asymmetric, $p / d+\mathrm{A}$ collisions in the same sense and with the same accuracy as was done in $\mathrm{A}+\mathrm{A}$. We argue that high $p_{T}$ direct photons offer an a posteriori test of any method suggested to categorize $p / d+\mathrm{A}$ - and in general, very asymmetric - collisions: the method is only viable if the nuclear modification factor for high $p_{T}$ direct photons is about unity for all centrality classes.
\end{abstract}

The 26th International Nuclear Physics Conference

11-16 September, 2016

Adelaide, Australia

*Speaker.

${ }^{\dagger}$ A footnote may follow. 


\section{Introduction}

Establishing collision geometry from experimental observables when the internal substructure of the colliding objects is relevant, is an old problem in particle and nuclear physics. Here "collision geometry" can be something as simple as the impact parameter $b$, or the shape of the overlap region of the two objects (particles or nuclei), the spatial distribution of the components (partons or nucleons) therein, the number of components in the two objects that participate in some interaction (parton or nucleon participants, $N_{\text {part }}$ ), finally the total number of such interactions ("binary collisions", $\left.N_{\text {coll }}\right)$. Since none of these are directly observable, the transition from $b$ to $N_{\text {part }}, N_{\text {coll }}$ requires some theoretical model. The issue was first addressed on the nucleon (hadron) level by Glauber [1]. His model assumes incoherent collisions of the nucleons moving on a straight path, constant $\sigma_{N N}$ and small momentum transfer in the individual $N N$ collisions ("...the approximate wave function (74) is only adequate for the treatment of small-angle scattering. It does not contain, in general, a correct estimate of the Fourier-amplitudes corresponding to large momentum transfer"). Despite these limitations the Glauber-model is successfully used in relativistic heavy ion collisions to establish $N_{\text {part }}, N_{\text {coll }}$ and to connect the impact parameter $b$ (or "centrality") to experimentally measurable quantities - at least when the colliding ions are both large. The procedures are described for instance in [2] including the caveat that "in heavy ion collisions we manipulate the fact that the majority of the initial state nucleon-nucleon collisions will be analogous to minimum bias $p+p$ collisions". Recently is has been observed [3] that bulk observables, like $d E_{T} / d \eta$ are better described if the fundamental interactions happen between constituent-quarks rather then nucleons, but this approach still preserves the basic characteristics of the Glauber model (incoherent scatterings, straight path, constant $\sigma_{q q}$ ). If $N_{q p}$ is the number of constituent quark participants, the mean transverse energy $\left\langle d E_{T} / d \eta>/ N_{q p}\right.$ is approximately constant for a wide range of collision energies, at least when the colliding ions are both large. Even if there are occasional large fluctuations in an individual nucleon-nucleon (or parton-parton) scattering, their effect is washed out by the large number of average collisions between constituents in the event.

The idea of fluctuating cross-sections has been introduced by Gribov [4] and gained traction as collision energies increased and also with the study of asymmetric systems - $p+\mathrm{A}$ at first, followed by light-on-heavy ion collisions. One of the first examples was the series of transverse energy measurements by the NA34 (HELIOS) collaboration using ${ }^{32} \mathrm{~S}$ beams on various targets, from $\mathrm{Al}$ to $\mathrm{U}[5,6]$. With increasing target size the tails of the $E_{T}$ distributions exceeded more and more the expectations from independent nucleon-nucleon collisions; the excess was attributed to fluctuations in $E_{T}$ production, characterized by an empirical parameter $\omega$ that increases monotonically with target size. This parameter has been tied to cross-section fluctuations in [7], due to the (frozen) initial configuration of the nucleons. This opened the way to a modification of the original Glaubermodel, including the calculation of $N_{\text {coll }}$. However, experiments at RHIC and LHC continued using the original Glauber-model to determine collision centrality, $N_{\text {part }}$ and $N_{\text {coll }}$ until the early 2010s. It is interesting to note that in the 2007 review paper on "Glauber Modeling in High Energy Nuclear Collisions" [2] cross-section fluctuations are not discussed yet, not even as a footnote. This situation quickly changed once experiments at RHIC and LHC started to take large amounts of $\mathrm{p} / \mathrm{d}+\mathrm{A}$ data, originally meant to fine-tune our understanding of the initial state, impact-parameter dependent nuclear PDFs, and cold nuclear matter effects in general. Instead, some very unexpected 
results were found, primarily for the centrality dependence of nuclear modification factors, which left only two (not mutually exclusive) possibilities. Either some new physics processes, so far not seen, have to be considered - or we have to re-think how collision geometry can be determined from experimental observables.

\section{Centrality, nuclear modification factor $-\mathbf{A}+\mathbf{A}$ collisions}

In heavy ion experiments the collision geometry is usually implied from some global observable, like charged particle production $\left(N_{c h}\right)$, total transverse energy $\left(E_{T}\right)$, sometimes also by counting the number of nucleons (often $n$ only) that didn't take part in any interaction (spectators). Both $N_{c h}$ and $E_{T}$ are dominated by particles coming from soft interactions. The measurement is usually (but not always) done far away in (pseudo)rapidity from the region where the centrality-dependent signals will be studied, in order to minimize auto-correlations. The total distribution is then divided up to percentiles, providing the "centrality" classification of the event. The connection to the directly inaccessible $b, N_{\text {part }}, N_{\text {coll }}$ is then made with a Glauber-model based Monte Carlo [2]. For each participating nucleon (quark) the contribution is modeled with a negative binomial distribution (NBD), tuned such that its convolution with the calculated $N_{\text {part }}$ distribution reproduces the measured $N_{c h}$ or $E_{T}$. Simulations show that the method works well when large ions collide: the correlation between $N_{c h}$ and $N_{\text {part }}$ (and $b$ or $N_{\text {coll }}$ ) is tight. This is also confirmed by the nuclear modification factors.

In general terms the nuclear modification factor for an observable $X$ (particle species, jet) and nuclei $A, B$ is defined as

$$
R_{B A}^{X}=\frac{d N_{B A}^{X} / d p_{T} d y}{<N_{\text {coll }}>d N_{p p}^{X} / d p_{T} d y}
$$

i.e. the ratio of the yield observed in the heavy ion collision and the yield in $p+p$ scaled by the average number of (binary) nucleon-nucleon collisions. If $R_{B A}^{X} \approx 1$ it is usually interpreted as the absence of any specific nuclear (or medium) effects - although this is clearly a necessary condition only, not a sufficient one. If a strongly interacting medium is formed in the collision, the partons are expected to lose energy therein, making $R_{B A}^{X}<1$ at higher $p_{T}$, where hard scattering (i.e. early) processes are expected to be the dominant production mechanism. Such suppression has indeed been observed for various hadrons and jets at all RHIC and LHC experiments. Of course the absolute value of $R_{B A}^{X}$ depends on $N_{\text {coll }}$, calculated from a Glauber-model, which in turn is connected with the experimental centrality by soft particle production, in a different rapidity region, also, depending on $N_{\text {part }}$ rather than $N_{\text {coll }}$.

Validation of this procedure was ultimately provided by high $p_{T}$ direct photons, predominantly produced in initial hard scattering, but then, being color-neutral and with $\alpha_{e m}<<\alpha_{s}$ they are passing through the colored medium virtually unaffected. This way they are a good candidate to "calibrate" the number of hard collisions, and, by extension, the $N_{\text {coll }}$ calculated from the Glauber model. Before showing this, it is worth noting, that their production in $p+p$ is theoretically well understood (see Fig. 6 in [9]). The $x_{T}$ scaling of the experimental data published until 2012 is shown in Fig. 1 reproduced from [8]. Over two orders of magnitude in $x_{T}, 13$ orders of magnitude in cross-section and a factor of 350 in $\sqrt{s}$ all data (with the exception of the controversial Fermilab E706 results) line up on a single curve, from which an exponent $n=4.5$ can be derived. Leading 
order $(2 \rightarrow 2)$ processes only would result in $n=4$; the small deviation from this value indicates that higher order processes don't contribute substantially to the photon yield.

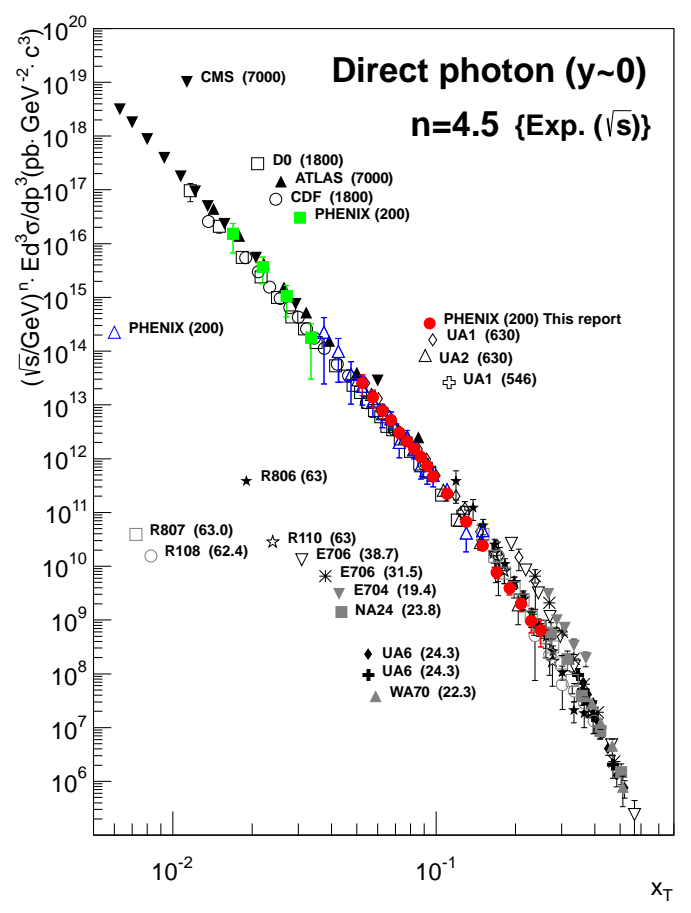

Figure 1: Various direct photon cross section measurements in $p+p$ and $p+\bar{p}$ collisions scaled by $(\sqrt{s})^{4.5}$ vs $x_{T}=2 p_{T} / \sqrt{s}$. The legend shows the experiment and the center-of-mass energy $(\mathrm{GeV})$ in parenthesis. References can be found in [8].

As it is well known, in heavy ion collisions all RHIC and LHC experiments found that in A+A high $p_{T}$ hadrons are suppressed, $R_{A A}^{\text {had }}$ - calculated with the Glauber $N_{\text {coll }}$ - is strongly dependent on centrality, and usually $R_{A A}^{\text {had }}<<1$. In stark contrast, for photons $R_{A A}^{\gamma} \approx 1$ was observed for all centralities (see for instance [10,11, 12]). Since $R_{A A}$ has been calculated with the same $N_{\text {coll }}$ both for hadrons (suppression observed) and photons (suppression neither expected nor observed), this is a potent validation of the Glauber model in $\mathrm{A}+\mathrm{A}$.

It should be noted that strictly speaking $R_{A A}^{\gamma}$ should not necessarily be unity; there are at least three processes that can slightly modify it. The first is photons from jet fragmentation, where the parent partons already lost energy in the medium; however, these are only a small fraction of the photons [8] and often can be tagged by isolation cuts [12]. The second is called jet-photon conversion [13], when a fast quark passing through the sQGP produces photons by Compton scattering with the thermal gluons or annihilation with the thermal quarks. Photons from this process, originally thought to be the dominant source up to $5-6 \mathrm{GeV} / c$, are hard to tag experimentally, but if their rate is really that high, they should be identifiable from double jet-conversion of back-to-back hard scattered partons. The third modifying factor is the isospin effect in nucleus-nucleus collisions [14]. When calculating $R_{A A}^{\gamma}$ one scales the $p+p$ cross-section with $N_{\text {coll }}$ of all nucleons, but the photon production from $p+p, p+n$ and $n+n$ is different $\Sigma Q_{q}^{2}$ quark charge square sum of protons 
and neutrons. Despite these three caveats it is safe to say that $1 /$ production of high $p_{T}$ photons in $p+p$ is well understood, $2 / R_{A A}^{\gamma}$ is close to the expected value in large systems (consistent with unity within experimental uncertainties) $3 /$ while more precise measurements may reveal small deviations, those appear to be calculable. In short, high $p_{T}$ photons are "standard candle", a tool to calibrate $N_{\text {coll }}$.

\section{Centrality, nuclear modification factor $-\mathrm{p} / \mathrm{d}+\mathrm{A}$ collisions}

While hadron and jet suppression in A+A could be explained with the formation of an sQGP medium (final state effect only), other observations suggested that the initial (pre-collision) state can also be modified. The large data sets collected since 2008 at RHIC $(d+\mathrm{Au})$ and later at LHC $(p+\mathrm{Pb})$ were originally meant to study these initial state effects: the expectation was that colliding these very asymmetric systems (no more than two nucleons on a large ion) will probe the properties of the "cold" nucleus, where no sQGP is formed, and the results serve as a baseline in the study of medium effects in $\mathrm{A}+\mathrm{A}$. The centrality of the $p / d+\mathrm{A}$ collisions was initially determined by the same methods that worked well for A+A.

The first results were quite surprising. Observations of long-range azimuthal correlations and strong azimuthal anisotropies (flow) raise the possibility that even in these very asymmetric collisions droplets of sQGP can be formed. As for the nuclear modification factor $R_{p A}$ at mid-rapidity, the findings were even more puzzling. On the one hand, in "central" collisions $R_{p A}$ was suppressed (this in itself was still consistent with droplets of sQGP), on the other hand in "peripheral" collisions $R_{p A}$ showed significant enhancement $[16,17]$, defying all expectations and not seen in any previous $R_{A A}$ measurement. While some new physics mechanism producing such enhancement at high $p_{T}$ could not be excluded, in Occam's spirit it was only logical to assume that maybe the way centrality is determined in A+A isn't directly applicable in those very asymmetric collisions [18].

The strictly empirical argument in [18] was this. The only case where the correlation between soft production at high rapidities (where centrality is usually determined) and high $p_{T}$ particle/jet production at mid-rapidity can experimentally be verified without any bias or prior assumption is the case of $p+p$ collisions. In Fig. 2 taken from [15] this correlation is presented. On the left panel the average $N_{c h}$ in the forward detector (determining centrality and serving as trigger) is shown $v s$ the transverse momentum of the highest $p_{T}$ particle observed at midrapidity. The right panel shows the trigger efficiency $v s$ the same quantity. While the presence of a high $p_{T}$ particle at midrapidity causes only minor losses in the trigger efficiency, the depletion in forward $N_{c h}$ is very substantial and grows rapidly with $p_{T}$. This is not an issue in $\mathrm{A}+\mathrm{A}$ collisions, since there are many collisions between different nucleons, and even if one nucleon-nucleon scattering is hard, contributing less to the $N_{c h}$ at high rapidity, the deficit is virtually invisible since all other nucleon-nucleon collisions produce the average $N_{c h}$, and the centrality calculated with the Glauber model remains unbiased. The same is not true in $p / d+\mathrm{A}$ collisions: once the projectile suffers a hard collision, $N_{c h}$ is necessarily depleted. Even if its unaffected constituents have further interactions with nucleons in the target A, the total $N_{c h}$ will shift to lower values. As a consequence, an event with a high $p_{T}$ particle will be classified on the average as more peripheral than it actually is. This simple, qualitative picture has the advantage that it relies only on actual experimental observations, and it is consistent both with the apparent suppression of $R_{p A}$ in "central" and its apparent enhancement 

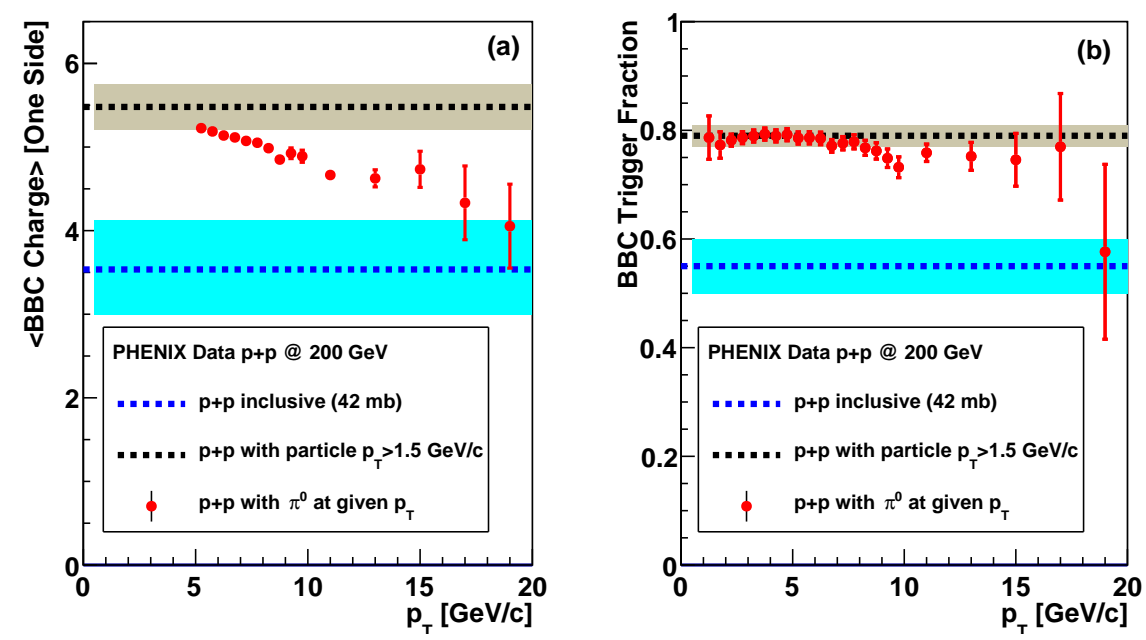

Figure 2: Left (a): $N_{c h}$ at $-3.9<\eta<-3.1$ vs the highest $p_{T}$ observed in a single particle at $|\eta|<0.35$ in $p p$ collisions [15]. The two dashed lines are the mean charge for events taken with minimum bias trigger (lower, blue) and requiring at least one particle with $p_{T}>1.5 \mathrm{GeV} / c$ at midrapidity. Right (b): Trigger efficiency (probability of the coincidence of at least one particle at both $-3.9<\eta<-3.1$ and $3.1<\eta<3.9$ ) for minimum bias events (lower, blue line), events with at least one particle with $p_{T}>1.5$ at midrapidity (upper, black line), and the dependence on the highest $p_{T}$ particle observed at midrapidity.

in "peripheral" $p / d+A$ collisions. It doesn't claim to provide an explanation of the underlying physics mechanisms.

In the past few years there were many attempts to modify the Glauber procedure for very asymmetric collisions based on some phenomenological model. In an early paper the Gribovpicture and the notion of color fluctuations has been re-introduced [19]. The authors found that in $p+$ A collisions "standard procedures for selecting peripheral (central) collisions lead to selection of configurations in the projectile which interact with smaller (larger) than average strength". The authors of [20] explicitely studied $N_{\text {coll }}$ in case of hard triggers and assuming the "flickering" of the interaction strength in $p+\mathrm{A}$ collisions, finding that "measurements by CMS and ATLAS for jets carrying a large fraction of the proton momentum, $x_{p}$, is consistent with the expectation that these configurations interact with the strength that is significantly smaller than the average one". The authors of [21] provide a model in which the removal of a large $x$ parton (the one producing the hard scattering) reduces the production of small $x$ partons by splitting, which in turn are responsible for soft production, leading to a kinematic depletion of soft particles if hard scattering occured in the event. Similar to cross-section fluctuations, in [22] the notion of weakly interacting or "shrinking" nucleon is explored to explain events with a high $p_{T}$ jet present, and predictions are made for centrality-dependent jet yields in $p+\mathrm{Au}, d+\mathrm{Au}$ and ${ }^{3} \mathrm{He}+\mathrm{Au}$ collisions at RHIC energies.

Some heavy ion experiments in the meantime tried to modify their Glauber calculations with bias factors [15], or published $R_{p A}$ with different $\omega$ parameters of the Glauber-Gribov model [23]. The ALICE experiment chose a different path by publishing nuclear modifications in terms of the purely experimental "event activity" rather than turning it into event geometry using a model that is not directly verifiable. 


\section{Direct photons to the rescue?}

Let us summarize our findings. The Glauber model and the centrality determination based on it works well when two large ions collide. This is not surprising: even if a few nucleons suffer "extreme" collisions, the regular soft particle production from the average binary collisions dominates the event (see also [2] and the correlations in [18]). Also, production of high $p_{T}$ direct photons is well understood in $p+p$ at all available energies. Finally, independent of centrality, the high $p_{T}$ photon $R_{A A}^{\gamma}$ is consistent with unity in $\mathrm{A}+\mathrm{A}$, modulo some small (and experimentally distinguishable) effects, listed earlier.

Now let us assume that the physics mechanisms in $\mathrm{A}+\mathrm{A}$ are a superset of the mechanisms in $p+\mathrm{A}-$ there is no new physics in $p+\mathrm{A}$ that wouldn't be present in $\mathrm{A}+\mathrm{A}$, albeit possibly suppressed by much larger effects present only in $\mathrm{A}+\mathrm{A}$. It then follows, that if photons prove to be a "standard candle" in A+A, they will be standard candle in $p+\mathrm{A}$, too. So far all measurements indicate that photons indeed are standard candle in $\mathrm{A}+\mathrm{A}$, their yield is not modified from the expected one in those cases, where centrality (and $N_{\text {coll }}$ ) is unambiguous. If so, then there is little reason to assume that $R_{p A}^{\gamma}$ will be modified (differ from unity) in $p+\mathrm{A}$.

This provides an opportunity to test a posteriori any model or procedure aimed to provide geometry $\left(N_{\text {coll }}\right)$ related information in $p+\mathrm{A}$ collisions. The lithmus test is whether the photon nuclear modification factor $R_{p A}^{\gamma}$ calculated with it is consistent with unity - for all centralities and in the entire high $p_{T}$ range - or not. Note that in light of Fig. 2 this second condition is also very important. If $R_{p A}^{\gamma}$ deviates from unity significantly in any direction, the model is very likely biased. Clearly, our test doesn't provide any guidance how to contruct geometry/centrality models or procedures. However, it gives a decisive test whether they are viable or not.

\section{References}

[1] Glauber R J, in Lectures in Theoretical Physics, ed Brittin W E, Dunham L G, 1:135. New York: Interscience (1959)

[2] Miller M L, Reygers K, Sanders S J, Steinberg P, Annu. Rev. Nucl. Part. Sci 57 (2007) 205

[3] Adler S S et al. (PHENIX Collaboration) Phys. Rev. C 89044905 (2014)

[4] V. N. Gribov, Soviet Physics JETP 30 (1970) 709

[5] Schukraft J (for the NA34/HELIOS Collaboration) Nucl. Phys. A498 (1989) 79c

[6] Akesson T et al. (NA34/HELIOS Collaboration) Nucl. Phys. B353 (1991) 1

[7] H. Heiselberg, G. Baym, B. Blättel, L. L. Frankfurt, M. Strikman, Phys. Rev. Lett. 67 (1991) 2946

[8] Adare A et al (PHENIX Collaboration) Phys. Rev. C 86, 072008 (2012)

[9] Aurenche, P, Guillet J Ph, Pilon E, Werlen M, Phys. Rev. D 73, 094007 (2006)

[10] Afanasiev S et al (PHENIX Collaboration) Phys. Rev. Lett. 109152302 (2012)

[11] Chatrchyan S et al (CMS Collaboration) Phys. Lett. B 710 (2012) 256

[12] Aad G et al (ATLAS Collaboration) Phys. Rev. C 93, 034914 (2016)

[13] Fries R J and Muller B, Phys. Rev. Lett. 90132301 (2003) 
[14] Arleo F, J. High Energy Phys. 09 (2006) 015

[15] Adare A et al. (PHENIX Collaboration), Phys. Rev. D 86072008 (2012)

[16] Aad G et al (ATLAS Collaboration) Phys. Lett. B 748, 392 (2015)

[17] Adare A et al, (PHENIX Collaboration) Phys. Rev. Lett. 116, 122301 (2016)

[18] G. David, Journal of Physics: Conference Series 589 (2015) 012005, arXiv:

[19] Alvioli M and Strikman M, Phys. Lett. B 722347

[20] Alvioli M et al, Phys. Rev. C 90034914 (2014)

[21] Bzdak A et al Phys. Rev. C 93044901 (2016)

[22] McGlinchey D et al Phys. Rev. C 94024915 (2016)

[23] Aad G et al (ATLAS Collaboration) Phys. Lett. B 763 (2016) 313 